\section{Sensitive training through body awareness to improve the writing of patients with writer's cramp}

\author{
Flavia Quadros Boisson Waissman,1,2 \\ Marco Orsini,1,3 \\ Osvaldo J.M. Nascimento, 1 \\ Marco Antônio A. Leite, 1 \\ João Santos Pereira1,2 \\ 1Neurology Department, Fluminense \\ Federal University - UFF, Niteroi; \\ 2Movement Disorders Section, Neurology \\ Service, Pedro Ernesto University \\ Hospital, The Rio de Janeiro State \\ University - UERJ; 3Science Reabilitation, \\ University Center Augusto Motta - \\ UNISUAM, Bonsucesso, Brazil
}

\section{Abstract}

The aim of this article is to evaluate if body awareness is important to improve the writing ability before using the splints. Twelve patients with writer's cramp were evaluated by a specialist in movement disorders. The BurkeFahn-Marsden Scale (BFM), the Jedynak Protocol and the Analog Pain Scale were used. The rehabilitation training was administered over an eight-week period, in two phases of four weeks each, twice a week. Each session would last 60 minutes. A significant improvement was shown, not only in the motor, but also in the pain perception, through the BFM scale, the Jedynak Protocol and the Analog Pain Scale. Therefore, we conclude that the motor training, thanks to the body awareness and immobilization by splints of the affected dystonic musculature, led to an important improvement in the writer's cramp writing.

\section{Introduction}

Focal dystonia is a specific-task disorder with difficult recovery, which occurs due to motor programming dysfunction, resulting in an imbalance between agonist and antagonist musculature during the movement. ${ }^{1}$ Many professionals such as teachers, writers, musicians and typists, among others, can develop the symptoms. ${ }^{2,3}$

Although the prevalence is relatively low, ranging from 3 to $7 / 100.000$, the writer's cramp can be scary if considered impaired work, persistent pain, inability to carry out activities, the decrease of self-esteem and socioeconomic commitment. A large body of evidence points to a role of basal ganglia dysfunction in the pathophysiology of dystonia (writer's cramp), but recent studies indicate that cerebellar dysfunction may also be involved. The cerebellum influences sensorimotor adaptation by modulating sensorimotor plasticity of the primary motor cortex. The loss of cerebellar control over sensorimotor plasticity might also lead to building up an incorrect motor program to specific adaptation tasks such as writing. ${ }^{4}$

The use of therapeutic medication that works via chemical blockage by local use of botulinum toxin has been the therapy normally used for writer's cramp (dystonia) management. Recent studies emphasize mechanical immobilization through the use of splints, with similar results. 5,6

The aim of this study was to applied a conservative therapeutic program through the immobilization of the affected muscles. Patients were instructed to identify the posture compensation, ${ }^{7}$ resulting from dystonia after the body awareness process using the splint.

\section{Materials and Methods}

Of a total of 21 volunteers with the clinical diagnosis of writer's cramp seen and examined in the Sector of Movement Disorders, Neurology at Pedro Ernesto University Hospital (HUPE), The Rio de Janeiro State University (UERJ), 12 were selected to participate in this study: seven men and five women, aged between 24 and 76, regardless of how long they have had the disease or level of dystonia, as long as they would obey the criteria of inclusion established i.e., not participating in any physiotherapy program, not making used of botulinum toxin for at least six months or using any medicine for dystonia treatment. The others were excluded for failing to meet the selection criteria pre-established, as six out of these were using anticholinergic medication and/or benzodiazepine besides recent botulinum toxin application. Other two with carpal tunnel syndrome pain that hindered daily activities and the last with intense referred shoulder pain were excluded.

Out of the 12 individuals, eleven were righthanded and one was left-handed. Their formal education ranged from elementary (one), high-school (eight) and university degree (three), holding different professions. All the patients were clinically evaluated by a specialist in movements through a neurological test to detect the level of dystonic, and later submitted to a physiotherapeutic evaluation.

The study was carried out at Pedro Ernesto University Hospital, Rio de Janeiro State University, RJ, Brazil, and obeyed the ethical procedures, approved by the ethics committee,
Correspondence: João Santos Pereira, Setor de Distúrbios do Movimento, Serviço de Neurologia, HUPE, UERJ, Av. 28 de Setembro 87 - $2^{\circ}$ andar, Vila Isabel 20550-030, Rio de Janeiro, Brasil.

E-mail: jspereira.md@uol.com.br

Key words: writer's cramp, focal dystonia, rehabilitation, writing.

Contributions: the authors contributed equally.

Conflict of interests: the authors declare no potential conflict of interests.

Received for publication: 27 August 2013.

Revision received: 28 October 2013.

Accepted for publication: 29 October 2013.

This work is licensed under a Creative Commons Attribution NonCommercial 3.0 License (CC BYNC 3.0).

(C) Copyright F. Quadros Boisson Waissman et al., 2013

Licensee PAGEPress, Italy

Neurology International 2013; 5:e24

doi:10.4081/ni.2013.e24

having all the participants sign an informed consent.

As employed by many studies, 1,8-14 the BFM, Jedynak Protocol and the Analogical Pain Scale, were the instruments used to evaluate the functional motor and pain.

A video camera (Sony, CCD-TRV 108 NTSC, Tokyo, Japan), was also used to record the patients during the initial and final evaluation, registering the dystonic postures as well as enabling a more detailed comparative analysis of the movements. This recording served to analyze the finger, hand, and forearm movements, visualizing and comparing the abnormalities during writing.

The electroneuromyography (EMG) could not be carried out on that occasion due to technical problems and the refusal of most of the participants. As clinical diagnosis was evident and all selected subjects had previously undergone EMG more than once, showing discrete changes in motor conduction or even without evidence of any disturbance, it was decided, for humanitarian reasons, not to do the exam above, because none of the participants wanted due to the previous results, which really should not be discarded, especially in individuals who had never performed such examination.

As the objective of the body awareness is to make the patient understand and recognize the cause of the problem, specific exercises were prepared for the elbow, wrist and finger articulations to improve the stability of the scapular girdle and thus, improve the fine movement of the hand. Such exercises were 
performed 20 minutes after relaxation and stretching when the patient would find himself in a calm, air-conditioned environment, listening to instrumental music, low illumination and lying on a bed for complete relaxation, which contributes to the improvement of dystonic disorders in the affected area.

The rehabilitation training was performed over 8 weeks, in two phases of 4 weeks each, twice a week for 60 minutes. In the first phase, body awareness with postural correction using relaxation techniques and sensory information were emphasized. In the second phase adequate splinting was used on the primary dystonic finger to inhibit the dystonic action while the patient was writing. Similar home exercises were set for 30 minutes daily.

The body awareness exercises conducted in the first phase of treatment were important for relaxation and awareness of motor dysfunction. During such activities, the patients were asked to make the pressure in the fingers conscious, the presence of muscle tension and the relationship between each body segment and its relation to space. This postural reeducation and gestural schemes were based on studies of Chamagne et al. ${ }^{7}$ and Byl et al. ${ }^{2}$ that aims to restore physiological posture giving support to the natural gestures, being the last steps to return to normal daily activities.

The wrist immobilization has the objective to increase the sliding capacity of the forearm during the written exercises. In order to sustain and strengthen the shoulder muscles, the following exercise was carried out: draw circles on a paper holding the pen between the thumb and the index finger, maintaining the forearm elevated (without resting the elbow on the table). Then, the same exercise was made resting the elbow on the table. Here the objective was to differentiate the movements of the wrist from those of the shoulder. The importance of the practice was to help patient recognize the difference between writing on a paper and writing on a blackboard. Writing on paper uses the distal segment of the upper limb (hand), and the writing on a blackboard uses the proximal segment (shoulder).

During the exercises, the patient was oriented to become aware of the grip in the fingers of the muscular tension and the relationship of each body segment with each other. This postural re-education is similar to other studies. 2,9

Motor coordination and concentration training were stimulated through games such as domino, checkers etc. In the motor training phase the patient resumes his writing activities under supervision using specific splints (ring in eight, North Coast R) numbered according to the finger diameter of the hand presenting dystonia (Figure 1). Such phase is based on the fingers and wrist immobilization principles of the dystonic side through the splint choice and determination of training time, 1,5 in which one or more fingers are immobilized, depending on the dystonic pattern found (splints to prevent the fingers from flexing or extending) .

During four weeks, standard exercises were carried out for writing with the use of splints. The exercise consisted in covering dotted drawings, words, letters and numbers; copying paragraphs of a text and copying headlines from newspapers on white sheets of paper, being all writing held in cursive, forbidding the block letters, which facilitated motor performance. During training it was asked to write as many words as possible in steps of two minutes as, on average, this was the maximum time they could write continuously, although sometimes pain and muscle fatigue occurred. Motor activities were held throughout the training period. The size of the paper and the lines were defined in advance. During the rehabilitation process sheets of paper with lines or individual writing paper were used, since the overlapping of several sheets made writing easier by reducing the friction of the pen on the paper due to the pressure involved, working as an absorber. When using a notebook with several overlapping sheets of paper it was possible to indirectly measure the pressure of the pen on the paper, checking the number of sheets that were marked during the writing using the pen or pencil.

Before starting such writing activities, the patient could freely choose between thinner or thicker diameter pens, with or without rubbers or pencils, so that they would feel comfortable and the most functional possible. In some exercises color pencils or hydro color pens would be used.

Ending this phase, each participant was submitted to the same protocols initially applied to evaluate the motor activity and then a new filming was made.

\section{Statistics}

The comparison between objective and subjective parameters of the pre- and post-treatment moments was carried out through the Wilcoxon test. The meaningful statistic level chosen was $\mathrm{P}<0.05$.

\section{Results}

Twelve patients diagnosed with writer's cramp (7 male and 5 female) were evaluated; their mean age was 52, 17 \pm 16.07 (limit: 24$76)$. The average duration of the dystonia was $10.83 \pm 8.47$ years (limit: $2-38$ ). The initial diagnosis was done when the patients were at the age of $41.83 \pm 13.44$. The average time of the daily activity was $181.67 \pm 92.43$ minutes. Only $16.67 \%$ had a writer's cramp family history.
Figure 2A shows the writing characteristics obtained through the analysis of the pre- and post-treatment, using Burke-Fahn-Marsden (BFM) Scales and Jedynak Evaluation. By statistically evaluating the functional aspects through the Wilcoxon test, significant differences can be found between the pre- and posttreatment moments for all the variables of the BFM Scale (Table 1).

The assessment of muscle pain presented to writing through the analog scale of pain before and after treatment is described in Figure 2B. As far as the Analog Pain Scale goes, the Wilcoxon test showed that there are significant differences between the pre- and posttreatment for variables fist, forearm, arm and fingers. No significant differences were found among variables: tenar region, hypothenar and shoulder (Table 2 and Figure 2B). Table 3 shows the frequency of dystonic postural types observed during writing in pre- and post-treatment. The presence of more than one posture for each participant is interesting to observe.

Figure $2 \mathrm{C}$ shows the main affected muscles, considering the number of participants during the functional physiotherapeutic evaluation in pre- and post- treatment.

\section{Discussion}

Although known for some years, there is still difficulty in the diagnosis and treatment of writer's cramp. The need to know the clinical aspects of this disorder that affects the population in the productive phase, causing serious problems not only socio-economic but also psychological, remains challenging. Early diagnosis should be considered, so that preventive measures can be taken. This way, its identification in students who have bad posture is necessary, where harsh environmental condi-

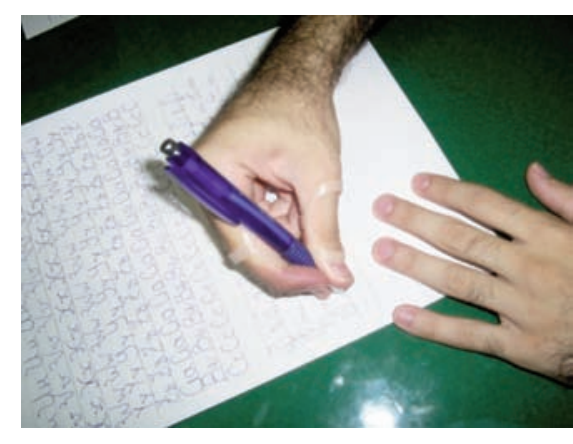

Figure 1. Patient during the motor training phase under supervision using specific splints numbered according to the finger diameter of the hand presenting dystonia. 
underestimated diagnosis.

The immobilization of muscles affected in the upper limb demonstrated significant improvement in focal hand dystonia during writing, as in previous studies. 5 The good handwriting quality was evident during training, as long as the writing speed was slow. However, under the stress required in rapid writing, the response was opposite. Despite having the writing speed reduced in this study, in another with musicians, such thing was not observed. In fact, there was an increase in speed. ${ }^{16}$ In our sample we could observe that the average age was high, with an intense degree of motor impairment, which made it difficult, but it did not impede the therapy.

There are few non-pharmaceutical studies for the writer's cramp treatment. The use of

Table 1. Evaluation of the results obtained by BFM Scales and the Jedynak Writing Evaluation of pre- and post-treatment using the Wilcoxon test $(n=12)$.

\begin{tabular}{|c|c|c|}
\hline Variables & $\mathbf{Z}^{*}$ & $\mathrm{P} * *$ \\
\hline PFSF post - PFSF pre & -2.714 & 0.007 \\
\hline WI post - WI pre & -3.207 & 0.001 \\
\hline WL post - WL pre & -3.276 & 0.001 \\
\hline DQ post -DQ pre & -2.486 & 0.013 \\
\hline
\end{tabular}

X Severity Factor; WI, Writing Incapacity; WL, Writing Legibility; DQ, Drawing Quality.

Table 2. Comparison of results obtained using the Analogue Pain Scale in the pre- and post-treatment, through the Wilcoxon test for body area affected.

\begin{tabular}{lcc} 
Variables & $Z^{*}$ & $\mathrm{P}^{* *}$ \\
Finger post - Finger pre & -2.413 & $0.016^{*}$ \\
Tenar region post - Tenar region pre & -1.693 & 0.090 \\
\hline Hyphotenar post - Hyphotenar pre & -1.604 & 0.109 \\
Wrist post - Wrist pre & -2.371 & $0.018^{*}$ \\
\hline Forearm post - Forearm pre & -2.527 & $0.012^{*}$ \\
Arm post - Arm pre & -2.032 & $0.042^{*}$ \\
\hline Shoulder post - Shoulder pre & -1.473 & 0.141 \\
\hline
\end{tabular}

*Statistic value $\mathrm{Z}$ to 1 degree of freedom and $\alpha<0.05$ established a priori. ${ }^{*}$ Statistically different to the level $\mathrm{P}<0.05$.

Table 3. Number of dystonic postural aspects observed during writing in pre and post treatment.

\begin{tabular}{lcc} 
Dystonic postural aspects & Pre & Post \\
Superposition of índex finger in relation to thumb & 1 & 0 \\
Extension of metacarpophalangeal joint of finger II & 1 & 0 \\
\hline Extension of fingers II, III and IV & 1 & 0 \\
Wrist extension with neutral position of forearm & 1 & 0 \\
\hline Extension of finger II & 2 & 0 \\
Flexion of distal phalange of the thumb with strong pressure on the paper & 6 & 1 \\
\hline Pinch of the pen between fingers II and III & 1 & 0 \\
Pinch of the pen between fingers I and III & 1 & 1 \\
\hline Ulnar deviation with pronation of forearm & 3 & 0 \\
Extension and radial deviation with pronation of forearm & 1 & 1 \\
\hline Radial deviation and wrist flexion with forearm in neutral position & 1 & 1 \\
Wrist dystonic tremor & 2 & 2 \\
\hline
\end{tabular}

splints as a therapeutic rehabilitation gained format at the beginning of this century with various studies. $16-18$

The rehabilitation program that lasted eight weeks showed improvement in focal hand dystonia. In spite of not being evident that it is the most adequate length of treatment it is, on

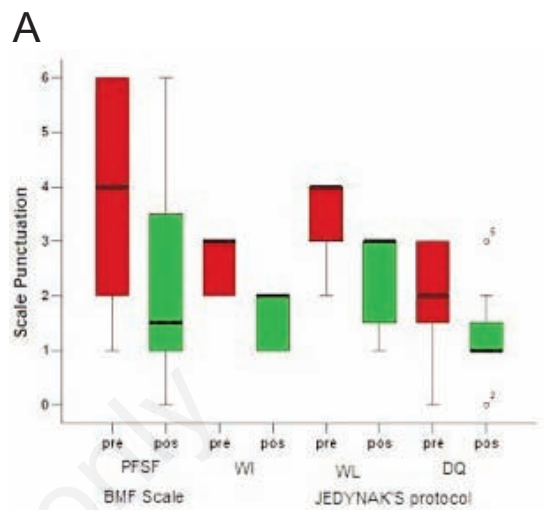

B

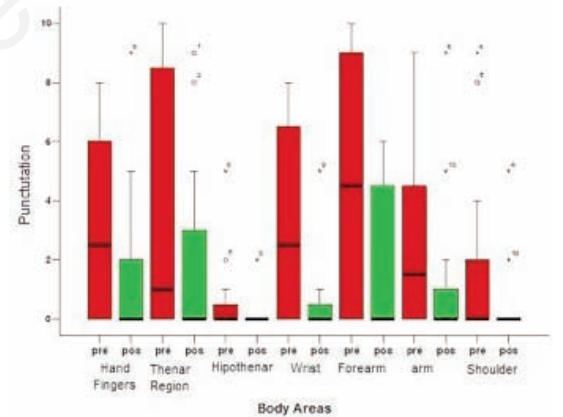

C

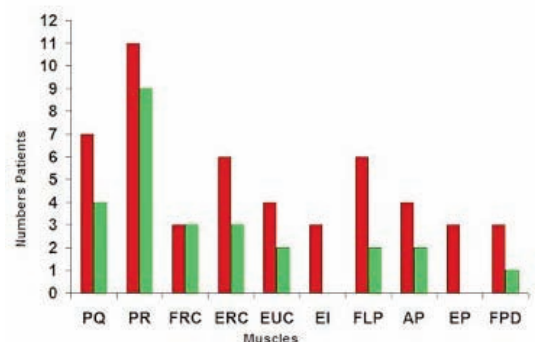

Figure 2. A) Analysis of the descriptive BFM scales and the Jedynak Writing Evaluation $(n=12) ; B)$ analysis of the descriptive measures found in the pain evaluation by body area, using the Analogical Pain Scale; C) relation of affected muscles per participant in pre- and post- treatment, during functional therapeutic evaluation. Red = pre-treatment; Green $=$ post-treatment. PFSF, Provocation Factor X Severity Factor; WI, Writing Incapacity; WL, Writing legibility; DQ, Drawing quality; $\mathrm{PQ}$, Pronator quadratus; PT, Pronato teres; CRF, Carp radial flexor; CRE, Carp radial extensors; CUE, Carp ulnar extensor; IE; Index extensor; FPL, Flexor pollicis longus; AP, Adductor pollicis; EP, Extensors pollicis; FDP, Flexor digitorun profundus. 
average, the most widely used. However when there is an interruption of practice and therapeutic assistance, followed by the guidance and monitoring of the patient, there is regression of the gain obtained during therapy.1,17,18

The body awareness was imperative for the good results obtained during the motor training of the writing. The option was for the BFM Scale because it is a widely used evaluation method, easy to follow-up. The Jedynak Protocol and the subjective Analog Pain Scale completed the evaluation process. 19

Improvement in the handwriting also occurred during the training, whenever the exercise was carried out slowly, which was not being observed in a stress situation that would demand quick writing. Baur et al.12 have found the same results by demonstrating that the patients were not able to write quickly. To improve the quality of the handwriting, the manual movements have to be carried out more slowly than normal, as previously described. 14

At wrist level, the pain was reduced on forearm and arm. This fact deserves a special attention because the patients with writer's cramp put too much pressure on the pen when holding it with the thumb and the index finger and at the same time using wrist and forearm. This occurs when there is a big difficulty in moving the forearm on the paper during writing (moving from left to right), provoking an involuntary and excessive muscular contraction on wrist and forearm resulting in pain. It was observed that the thenar region becomes very painful at the beginning of writing, immediately after starting. The positive results in diminishing the pain in the body areas, is due to previous body awareness, though the pain in the thenar region continued, but with less intensity. Pain on the shoulder was not consistently reported, nor the pain in the hypothenar region.

According to Baur et al. ${ }^{20}$ the absence of sliding of hand and forearm burdens the wrist resulting in slower writing and little legibility of handwriting. As it was impossible to objectively evaluate the pressure on the paper, the analysis was performed through direct visualization. As in some patients the pressure continued the same, the option was to maintain the conventional position and through body awareness modify the movements made. It was observed that the pressure clearly diminished, without the need to change the way of holding the pen. There was a direct relationship between pain and pressure on the paper, i.e., the more intense the pressure, the bigger the pain.

The evaluation of the pressure, the speed of writing and the counting of the number of letters can be done by computerized kinematic analysis of writing, using a tablet. ${ }^{18,21}$ It can also be observed the size and shape/type of let- ters. Self-assessment scales that describe the characteristics of the letters have been referred.1,11,18,21

An interesting fact was that most of the participants of this study opted for using a few sheets of paper, a minimum of two, instead of just one. According to them, they felt more confident this way. Schenk et al. ${ }^{21}$ point out that a focal dystonia is probably a poorly adapted compensation mechanism.

As observed in the present study, the immobilization of the affected musculature of the upper body members of the focal dystonia showed a sharp improvement in the motor performance. ${ }^{5,22}$ It was also observed that the dystonia factor was not necessarily related to persistent and intense act of writing, as some of the participants would write little during the day, while others have already stopped using the writing in their working activity or would not use writing in their activity at all, as the case of the professional shoe repairman who had the writer's cramp.

Through the subjective evaluation of the participants regarding the percentage of improvement on a scale of self-evaluation from 0 to $100 \%$, good results were obtained in terms of improvement of symptoms. Considering the percentage of $20 \%$ as the minimum, eight subjects had $60 \%$. A similar process was used by other authors in their reviews.1,2,17

Curiously, in a recent study, one of the patients, although having presented dystonia in writing for 12 years has also presented dystonia when using a spoon for eating, without presenting it in any other activity. A spreading of focal dystonia was suggested by Song et al. in 2008.23 This leads to the question and search for an answer of what is considered to be repetitive or stereotyped: whether it be a repetitive task done every day, even when performed for a short period of time, or if it is practiced for many hours during the day. Also of what can be considered short, average and long period of time.

As at the beginning the patient was very sensitive and anxious - maybe due to the long duration of his disability - a longer period of time was allocated to the playful activities so that he might get better aware of the inadequate posture of hand and thus, slowly regaining the movement of holding the pen between thumb and index finger. This helped the beginning of the writing training by favoring the right way to hold a pen.

The recovery of the affected task doesn't have to be necessarily directed to the writing activity, but can be directed to any physiological activity using the hand movement. Zeuner et al. 17 add to the already existing training the use of splints and therapeutic modeling clay to practice the finger movement. This reinforces the first phase of this study when games and activities were used to exercise the upper limbs affected by focal dystonia.

A common fact to some of the studies is the interference of the size of the pen used to write. ${ }^{24,25}$ The difference between using a pen or a pencil and even the presence of a rubber around the pen caused the increase of friction. Up to the present moment, these two observations have never been mentioned in any work before. For these patients, the pen as well as the pencil presented positive and negative aspects. The pen would slide more easily than the pencil helping the writing; the pencil however, allows the sensation of a better grip, but promotes more friction on the paper producing a thicker letter and would break more easily due to the big pressure made. Nevertheless, both instruments were used during the exercises according to necessity, whereas the pressure exercised by the patients would be observed.

As there is no standard conduct, it is difficult to perform an objective evaluation, as muscular fatigue and pain are completely subjective, depending on the patients' information. Furthermore, as posture is individual, it is characterized as a unique way of being. ${ }^{26,27}$

It was observed that $50 \%$ of the participants had an antagonist gesture and $25 \%$ involuntary movements in the contralateral hand during writing. Jedynak et al. ${ }^{11}$ showed that $44.6 \%$ of 65 patients showed these movements.

In a recent, study McKenzie et al..$^{28} \mathrm{com}$ pared the neuromusculoskeletal characteristics of those with writers' cramp (WC) and musicians' cramp (MC), and evaluate responsiveness to learning-based sensorimotor training. The study was composed of twenty-seven individuals (14 musicians, 13 writers) who participated for 8 weeks (supervised therapy), supplemented with a home program. Betweengroup differences on measures of musculoskeletal (physical), sensory, and motor performance were evaluated at baseline and postintervention. The results showed that patients with MC had a higher level of functional independence and better range of motion, but less strength in the affected upper limb than those of subjects with WC. MC patients demonstrated greater accuracy on kinesthesia, graphesthesia, and localization at baseline. No between-group differences in motor performance were noticed at baseline or post-intervention. Following individually adapted learningbased sensorimotor training, both groups improved in musculoskeletal (physical) parameters, sensory processing, and motor control; however, improvements on certain subtests differed by group. At follow-up, differences in posture, range of motion (ROM), strength, graphesthesia, and kinesthesia persisted between the groups.

Patients with WC should be seen as carriers of a specific form of dystonia with distinct pathophysiological framework in other ways. 
Treatment programs should be individualized, aiming the deficiencies and disabilities of patients.

In the case study we observed that conservative treatment, as carried out for writer's cramp, using splint to immobilize the finger compromised by focal dystonia in a young small guitar player, showed satisfactory results. ${ }^{29}$

\section{Conclusions}

According to the observations, it can be concluded that the practice of daily exercises could benefit a better rehabilitation. Prospective studies with clinical follow-up for some years could clarify this doubt, evaluating life quality and using a multidisciplinary team. In addition, we expect dystonia treatment to begin with proper diagnosis and classification, followed by an appropriate search for underlying etiology, and an assessment of the functional impairment associated with the dystonia. Despite the therapeutic approach usually limited to symptomatic therapy, it should be adequate to the needs of each individual in order to integrate him to society and provide better quality of daily life.

The conservative treatment through immobilization with splints of the musculature affected by focal dystonia in the writer's cramp, seems to be efficient in our cases. It is believed that this therapeutic, non-invasive procedure could be associated to the treatment with botulinum toxin and highlight a more adequate answer in the motor recovery of this disturbance. The results obtained in this study, favored by the two phases of the treatment, show that the body awareness can contribute to the motor recovery.

\section{References}

1. Zeuner KE, Shill HA, Sohn YH, et al. Motor training as treatment in focal hand dystonia. Mov Disord 2004;20:335-41.

2. Byl NN, Nagajaran S, McKenzie AL. Effect of sensory discrimination training on structure and function in patients with focal hand dystonia: a case series. Arch Phys Med Rehabil 2003;84:1505-14.

3. Bogey RA, Elovic EP, Bryant PR, et al. Rehabilitation of movement disorders. Arch Phys Med Rehabil 2004;85 Suppl 1:415 .

4. Hubsch C, Roze E, Popa T, et al. Defective cerebellar control of cortical plasticity in writer's cramp. Brain 2013;136:2050-62.

5. Priori A, Presenti A, Cappellari A, et al. Limb immobilization for the treatment of focal occupational dystonia. Neurology. 2001;57(3): 405-9.

6. Tas N, Karatas GK, Sepici V. Hand Orthosis as a writing AID in writer's cramp. Mov Disord 2001; 16: 1185-9.

7. Chamagne P. Functional dystonia in musicians: rehabilitation. Hand Clinic 2003;19: 309-16.

8. Cimatti Z, Schwartz DP, Bourdain F, et al. Time-frequency analysis reveals decreased high-frequency oscillations in writer's cramp. Brain 2007;130:198-205.

9. Burke RE, Fahn S, Marsden CD, et al. Validity and reliability of a rating scale for the primary torsion dystonias. Neurology 1985;35:73-7.

10. Molloy FM, Carr TD, Zeuner KE, et al. Abnormalities os spatial discrimination in focal and generalized dystonia. Brain 2003;126:2175-82.

11. Jedynak PC, Tranchant C, de Beyl DZ. Prospective clinical study of writer's cramp. Mov Disord 2001;16:494-9.

12. Baur B, Fürholzer W, Jasper I, et al. Effects of modified pen grip and handwriting training on writer's cramp. Arch Phys Med Rehab 2009;90:867-75.

13. Currà A, Agostino R, Dinapoli L, et al. Impairment of individual finger movements in patients with hand dystonia. Mov Disord 2004;19:1351-7.

14. Tinazzi M, Farina S, Bhatia K, et al. TENS for the treatment of writer's cramp dystonia: a randomized, placebo-controlled study. Neurology 2005;64:1946-8.

15. Defazio G, Abbruzzese G, Livrea P, Berardelli A. Epedemiology of primary dystonia. Lancet Neurol 2004;3:673-8.

16. Candia V, Schäfer T, Taub E, et al. Sensory motor retuning: a behavioral treatment for focal hand dystonia of pianists and gui- tarists. Arch Phys Med Rehabil 2002;83:1342-6.

17. Zeuner KE, Hallet M. Sensory training as treatment for focal hand dystonia: a 1-year follow-up. Mov Disord 2003;18:1044-117.

18. Zeuner KE, Peller M, Knutzen A, et al. motor re-training does not need to be task specific to improve writer's cramp. Mov Disord 2008;23:2319-27.

19. Rosenkranz K, Butler K, Williamon A, et al. Proprioceptive training in musician's dystonia and writer's cramp. Neurology 2007;22:304-15.

20. Baur B, Schenk T, Fürholzer W, et al. Modified pen grip in the treatment of writer's cramp. Hum Mov Sci 2006;25:46473.

21. Schenk T, Baur B, Steidle B, Marquardt C. Does training improve writer's cramp? J Hand Ther 2004;17:349-63.

22. Chaná-Cuevas P, Kunstmann-Rioseco C, Rodruíguez-Riquelme T. Distonía del guitarrista: tratamiento con reeducación sensorial. Rev Neurol 2003;37:637-40.

23. Song IU, Kim JS, Lee KS. Task-specific focal hand dystonia with usage of a spoon. Parkinsonism Relat Disord 2008;14:72-4.

24. Koller WC, Vetre-Overfield. Usefulness of a writing aid in writer's cramp. Neurology 1989;38:149-50.

25. Rhoad RC, Stern PJ. Writer's cramp - A focal dystonia: etiology, diagnosis and treatment. J Hand Surg 1993;18:541-4.

26. Merello M, Carpintiero S, Cammarota A, et al. Bilateral mirror writing movement (mirror dystonia) in a patient with writer's cramp: functional correlates. Mov Disord 2006;21:683-9.

27. Waissman $\mathrm{FQB}$, Pereira JS, Nascimento 0JM. A new therapeutic proposal for writer's cramp: a case report. Sao Paulo Med J 2010;128:96-8.

28. McKenzie AL, Goldman S, Barrango C, et al. Differences in physical characteristics and response to rehabilitation for patients with hand dystonia: musicians' cramp compared to writers' cramp. J Hand Ther 2009;22:172-81.

29. Waissman F, Pereira JS, Nascimento OJM. Therapeutic immobilization for small guitar player's dystonia: a case report. BMJ Case Reports 2009;2009:bcr07.2008.0432. 